\title{
$\mathbf{R}$
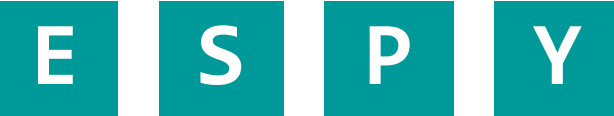 \\ N
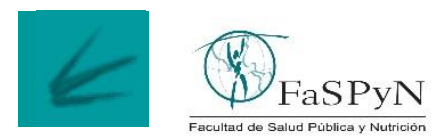

Revista Salud Pública y Nutrición

\section{EFICACIA DE LA INCORPORACIÓN DIETÉTICA DE ALIMENTOS BAJOS EN CARBOHIDRATOS SIMPLES Y ALTOS EN ANTIOXIDANTES SOBRE PARÁMETROS ANTROPOMÉTRICOS EN MUJERES CON SOBREPESO.}

\author{
EFFICACY OF DIETARY INCORPORATION OF LOW SIMPLE CARBOHYDRATE AND HIGH ANTIOXIDANT \\ FOODS ON ANTHROPOMETRIC PARAMETERS IN OVERWEIGHT WOMEN.
}

Arteaga-Murguia Dulce Margarita ${ }^{1}$, Alarcón-Domínguez Edith Efrén ${ }^{2}$, Gutiérrez-Sánchez Quetzali ${ }^{1}$, Rodríguez-Jiménez Hugo David ${ }^{1}$, Zamora-Gasga Victor Manuel ${ }^{3}$.

1 Universidad Vizcaya de las Américas. 2 Unidad de Medicina Familiar 18. Instituto Mexicano del Seguro Social. 3 Tecnológico Nacional de México, Tepic, Nayarit. México.

Citation: Arteaga-Murguia D.M., Alarcón-Domínguez E.E., Gutiérrez-Sánchez Q., Rodríguez-Jiménez H.D., Zamora-Gasga V.M. (2021) Eficacia de la incorporación dietética de alimentos bajos en carbohidratos simples y altos en antioxidantes sobre parámetros antropométricos en mujeres con sobrepeso. Revista Salud Pública y Nutrición, 20 (1), 20-29.

Editor: Esteban G. Ramos Peña, Dr. CS., Universidad Autónoma de Nuevo León, Facultad de Salud Pública y Nutrición, Monterrey Nuevo León, México. Copyright: (C2021 Arteaga-Murguia D.M., et al. This is an open-access article distributed under the terms of Creative Commons Attribution License [CC BY 4.0], which permits unrestricted use, distribution, and reproduction in any medium, provided the original author and source are credited.

Competing interests: The authors have declared that no competing interests exist.

DOI: https://doi.org/10.29105/respyn20.1-3

Recibido: 12 de mayo 2020; $\quad$ Aceptado: 30 de noviembre 2020

Email: vzamora@ittepic.edu.mx 


\title{
EFICACIA DE LA INCORPORACIÓN DIETÉTICA DE ALIMENTOS BAJOS EN CARBOHIDRATOS SIMPLES Y ALTOS EN ANTIOXIDANTES SOBRE PARÁMETROS ANTROPOMÉTRICOS EN MUJERES CON SOBREPESO.
}

\author{
Arteaga-Murguia Dulce Margarita ${ }^{1}$, Alarcón-Domínguez Edith Efrén ${ }^{2}$, Gutiérrez-Sánchez Quetzali ${ }^{1}$, Rodríguez- \\ Jiménez Hugo David ${ }^{1}$, Zamora-Gasga Victor Manuel ${ }^{3}$.
}

1 Universidad Vizcaya de las Américas. 2 Unidad de Medicina Familiar 18. Instituto Mexicano del Seguro Social. 3 Tecnológico Nacional de México, Tepic, Nayarit. México.

\section{RESUMEN}

Introducción. La dieta saludable es un factor determinante en la disminución de la incidencia de obesidad en la población mexicana, y la incorporación de vegetales y frutas a la dieta puede ser una alternativa para contrarrestar los efectos adversos de esta patología. Objetivo: Evaluar la eficacia de la incorporación dietética de alimentos bajos en carbohidratos simples y altos en antioxidantes sobre parámetros antropométricos en mujeres con sobrepeso del gimnasio municipal de Jalcocotán Nayarit. Material y Método: Se realizó un ensayo clínico cruzado aleatorizado. Nueve participantes fueron clasificadas en: Grupo experimental, consumió frutos, vegetales, vino tinto y fruto secos; Grupo control, siguió una dieta normocalórica. Al finalizar un mes, las dietas fueron cruzadas con un periodo previo de lavado de una semana. Se compararon las diferencias entre los parámetros antropométricos antes y después de la intervención (Prueba t student, $\alpha=0.05$ ). Resultados: La dieta experimental disminuyó significativamente el peso, índice de masa corporal, la circunferencia de cintura, cadera y brazo comparado con el control $(p<0.05)$. Discusión: Las frutas y los vegetales contiene menos carbohidratos simples y mayor contenido de fibra dietética que ha demostrado reducir la grasa abdominal, mientras tanto, antioxidantes como los polifenoles mitigan las complicaciones provocadas por la obesidad. Conclusiones: La sustitución de alimentos antioxidantes bajos en carbohidratos simples fue igualmente eficaz en la disminución del peso que una recomendación normocalórica.

Palabras Clave: Estrés oxidativo, sobrepeso, alimentos antioxidantes, fibra dietética, control del peso corporal.

\section{ABSTRACT}

Introduction: A healthy diet is a determining factor in the decrease in the incidence of obesity in the Mexican population, and the incorporation of vegetables and fruits into the diet may be an alternative to counteract the adverse effects of this pathology. Objective: The objective was to evaluate the effectiveness of dietary incorporation of foods low in simple carbohydrates and high in antioxidants on anthropometric parameters in overweight women from the Jalcocotán Nayarit municipal gym. Material and method: A randomized crossover clinical trial was performed. Nine participants were classified into: Experimental group, consumed fruits, vegetables, red wine and nuts; Control group followed a normal calorie diet. At the end of one month, the diets were crossed with a previous washing period of one week. The differences between the anthropometric parameters before and after the intervention were compared (Student's $t$ test, $a=0.05$ ). Results: The experimental diet significantly decreased weight, body mass index, waist, hip and arm circumference compared to the control $(p<0.05)$. Discussion: Fruits and vegetables contain fewer simple carbohydrates and higher dietary fiber content that has been shown to reduce belly fat, while antioxidants such as polyphenols mitigate obesity complications. Conclusions: The substitution of antioxidant foods low in simple carbohydrates was just as effective in weight loss as a normocaloric recommendation.

Key words: Oxidative stress, overweight, antioxidant foods, dietary fiber, body weight control. 


\section{Introducción}

En la actualidad, los patrones de consumo de la población adulta mexicana han indicado dietas de mala calidad con una progresiva inclusión de alimentos nocivos en las diferentes regiones del país (Pérez-Tepayo et al., 2020). En este sentido, Dosamantes-Carrasco et al. (2017)se ha establecido que una mayor adherencia a la ingesta de alimentos poco saludables aumenta el riesgo de desarrollar indicadores antropométricos no saludables y en consecuencia la incidencia de obesidad. En México, la prevalencia del sobrepeso y la obesidad y sus trastornos metabólicos asociados se consideran una gran amenaza para la salud pública que afecta principalmente a las mujeres. En 2018, se encontró que $36.6 \%$ de las mujeres de 20 años o más presentaron sobrepeso y $40.2 \%$ obesidad (INSP, 2018). En Nayarit, los resultados en mujeres de 20 años o más son similares a nivel nacional. La prevalencia de sobrepeso y obesidad fue de 35.6 y $36.5 \%$, respectivamente (INSP, 2012). La obesidad se caracteriza por una inflamación crónica de bajo grado con un estrés oxidativo permanentemente aumentado, esta sobreexpresión del estrés oxidativo daña las estructuras celulares junto con la producción insuficiente de mecanismos antioxidantes, lo que lleva al desarrollo de complicaciones relacionadas con la obesidad (Marseglia et al., 2015). En este sentido Amirkhizi et al. (2010) menciona que, la susceptibilidad al daño oxidativo es aún mayor en sujetos obesos debido a la disminución de las fuentes de antioxidantes, incluida la superóxido dismutasa, glutatión peroxidasa y catalasa, vitamina $\mathrm{A}$, vitamina $\mathrm{E}$, vitamina $\mathrm{C}$ y $\beta$-caroteno. La dieta y el ejercicio son claves en el tratamiento de estas patologías. Particularmente, los polifenoles de la dieta son una clase de fitoquímicos naturales, de los cuales algunos tales como catequinas, antocianinas, resveratrol y curcumina han demostrado modular las vías fisiológicas y moleculares que están involucradas en el metabolismo energético, la adiposidad y la obesidad (Tucakovic et al., 2015). Las dietas que incluyen una variedad de alimentos y componentes alimentarios como la fibra dietética y compuestos antioxidantes tienen efectos beneficiosos sobre los marcadores de enfermedad y los resultados de salud, incluida la regulación del peso corporal, importante en la prevención de enfermedades crónicas (Slavin y Green, 2007). El efecto del cambio en el perfil de alimentos consumidos sobre la pérdida de peso ha sido documentado en mujeres adultas por diversos autores, particularmente el incremento en la ingesta de verduras y frutas (Dreher y Ford, 2020). Svendsen et al. (2007) encontraron que el incremento en el consumo de verduras (hasta al menos $400 \mathrm{~g}$ / día) y frutas (hasta al menos $300 \mathrm{~g} /$ día) incrementa la pérdida de peso hasta $3 \%$ y aumenta las concentraciones plasmáticas de $\alpha$ - y $\beta$-caroteno. En un estudio retrospectivo de 6 meses, 66 mujeres obesas encontraron que el incremento en el consumo de verduras y frutas y la reducción del consumo de dulces y frituras fue relacionado con una disminución en el peso corporal medio en un 3.2\%, el IMC en 2.5 $\mathrm{kg} / \mathrm{m} 2$, la masa grasa corporal en un $1.7 \%$ y la circunferencia de cintura en $4.8 \mathrm{~cm}$ en comparación a los niveles de referencia (Aguiar-Bloemer et al., 2019). Sin embargo, aún no son claros los efectos de estas dietas en la reducción de los parámetros antropométricos. Los supuestos beneficios de estas dietas pueden atribuirse a mecanismos que involucran la regulación de la saciedad y el apetito, el control glucémico e insulinémico, la regulación de los lípidos y la reducción del estrés oxidativo y los procesos inflamatorios. Por lo tanto, el objetivo del presente trabajo fue evaluar la eficacia de la incorporación dietética de alimentos bajos en carbohidratos simples y altos en antioxidantes sobre los parámetros antropométricos en mujeres con sobrepeso que asisten al gimnasio municipal de Jalcocotán Nayarit, México.

\section{Material y Método}

$\underline{\text { Selección de los participantes }}$

La presente investigación se trató de un estudio analítico, longitudinal, prospectiva y experimental. La selección de la muestra se obtuvo por muestreo no probabilístico discrecional. La selección de los participantes se estableció utilizando los siguientes criterios de inclusión: Femeninas con sobrepeso (25 $\mathrm{kg} / \mathrm{m}^{2}<\mathrm{IMC}<29.9 \mathrm{~kg} / \mathrm{m}^{2}$ ) de 25 a 40 años, sin alguna patología agregada, que realizaban una hora de ejercicio en bicicleta estática a la semana, con un periodo de frecuencia entre 5 y 12 meses adscritas al Gimnasio municipal de Jalcocotán, Nayarit. Un total de nueve mujeres fueron elegidas previa autorización y firma de consentimiento informado. Se determinó la potencia de la prueba con el programa para análisis epidemiológicos de datos Epidat versión 4.1 mediante la fórmula de comparación de medias en dos grupos emparejados, considerando como variable de desenlace el peso. La potencia de la prueba fue de $33.8 \%$ considerando una diferencia de 
medias a detectar de 0.58 , una desviación estándar de las diferencias de 1 y un nivel de confianza del $95 \%$. El trabajo experimental fue realizado durante el periodo comprendido entre julio y septiembre del 2019.

Diseño de la investigación

El estudio fue un ensayo clínico aleatorio, simple ciego, cruzado de dos periodos (Ver Figura 1). convencionales de tipo normocalórica (AC). Los menús fueron ajustados utilizando la tasa metabólica basal (TMB) calculada con la fórmula de Harris Benedict (Roza y Shizgal, 1984), considerando un efecto termogénico de los alimentos del $10 \%$ y una actividad física moderada de $20 \%$. El rango de ingesta energética total de las pacientes fue entre 1742 y 1977 kcal. Ambas recomendaciones dietéticas se ajustaron al consumo de $20 \%$ de proteínas, $50 \%$ de carbohidratos y 30\% de lípidos de

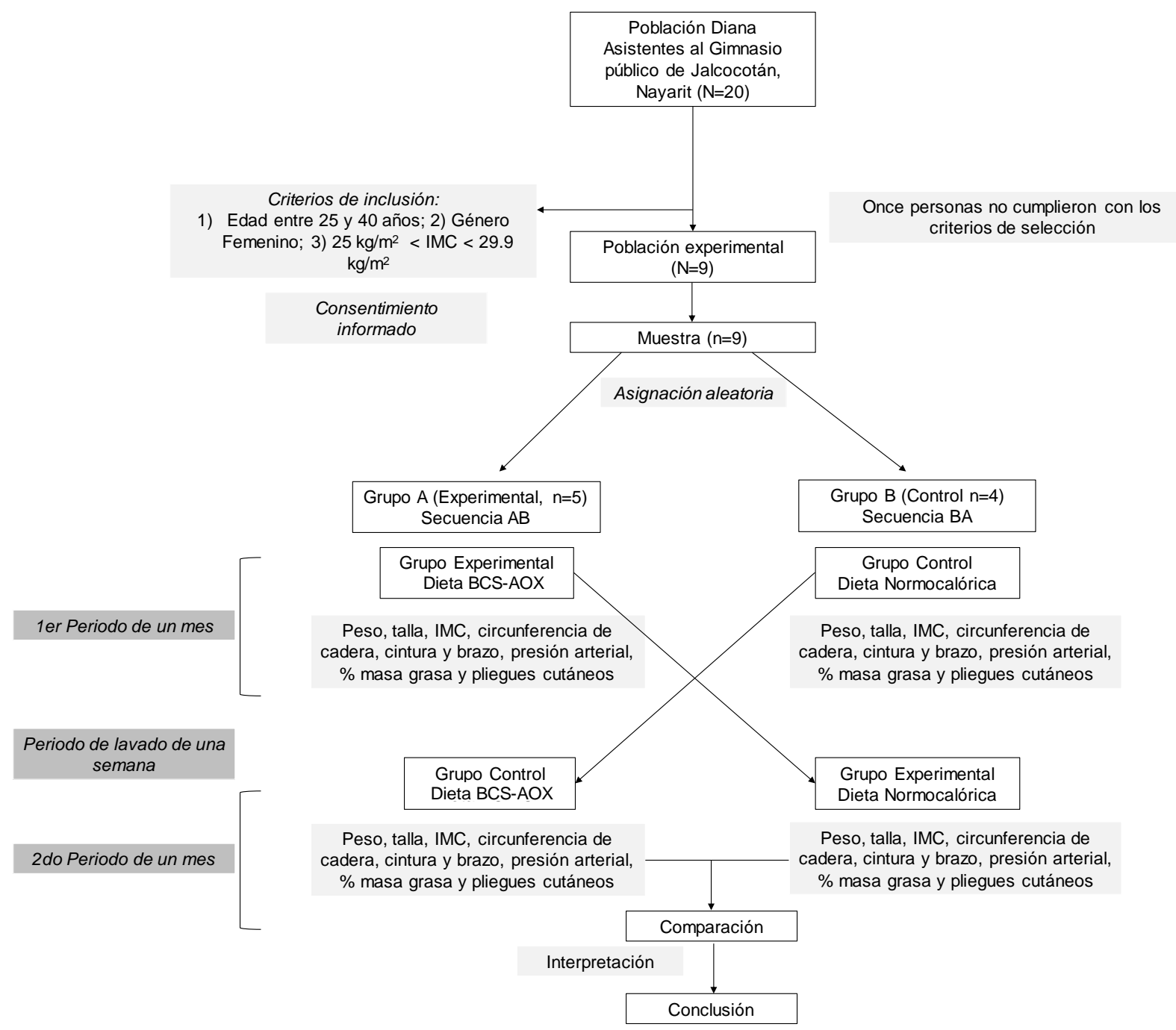

Durante cada periodo, los sujetos recibieron instrucciones para consumir menús de dos tipos de recomendaciones nutricionales, una con alimentos bajos en carbohidratos simples y altos en antioxidantes (BCS-AOX) y otra con alimentos la ingesta energética total. Sin embargo, en los menús de las recomendaciones nutricionales BCS-AOX, $25 \%$ de la ingesta total fue sustituida por la incorporación de alimentos considerados fuentes de antioxidantes (Polifenoles identificados $\geq 14$ según 
la Base de Datos Phenol Explorer versión 3.6, http://phenol-explorer.eu/) y/o bajos en carbohidratos simples (Carga glucémica $<10$ y/o índice glucémico < 50, según el Sistema Mexicano de Alimentos Equivalentes, SMAE) como verduras (calabaza, apio, tomate, coles, zanahoria, pimiento, espinaca y brócoli), frutas (cítricos, fresas, kiwi, melón, guayaba, uva roja, arándanos, frambuesas y moras), vino tinto y frutos secos. Las porciones fueron establecidas utilizando el SMAE (PérezLizaur et al., 2014) y la ingesta energética total fue distribuida durante el día en cinco tiempos de ingesta (Desayuno, primera colación, comida, segunda colación y cena). Ambos grupos recibieron orientación nutricional inicial referente al plato del bien comer y algunas patologías y consecuencias que se desarrollan cuando existe problemas de sobrepeso como la diabetes e hipertensión. Los sujetos desconocían las recomendaciones nutricionales a las que fueron asignados hasta que finalizó el estudio y se analizaron los datos. En el periodo inicial, cada participante fue asignado aleatoriamente para recibir las recomendaciones BCS-AOX o las de AC, que debían consumirse durante cuatro semanas. Este periodo inicial fue seguido por una fase de lavado de una semana, durante la cual los sujetos recibieron instrucciones de consumir los alimentos de acuerdo a su elección. Diferentes autores sugieren que un período de lavado de una semana fue suficiente para eliminar el efecto del tratamiento anterior cuando se trata de la suplementación de alimentos con alto contenido de compuestos antioxidantes (BarreraReyes et al., 2019; Roengrit et al., 2015). Finalmente, los sujetos fueron asignados para recibir el otro tratamiento por un período adicional de 4 semanas. Cuatro sujetos recibieron inicialmente las recomendaciones AC y cinco sujetos las recomendaciones de BCS-AOX. Las participantes mantuvieron sus rutinas de ejercicio durante la intervención. El cumplimiento del consumo de los alimentos en cada recomendación se calculó a partir recordatorios de alimentos en dos días no consecutivos cada 2 semanas.

Variables de estudio

A cada paciente se le aplicó una historia clínica que incluía la edad, ocupación, actividad física, escolaridad, estado civil y antecedentes patológicos heredofamiliares. Las evaluaciones antropométricas fueron realizadas por personal capacitado y se siguieron los protocolos establecidos por Suverza y
Haua (2010). Las variables evaluadas fueron peso $(\mathrm{kg})$, talla $(\mathrm{m})$ índice de masa corporal $(\mathrm{Kg} / \mathrm{m} 2)$, circunferencia de cintura, cadera y brazo $(\mathrm{cm})$, masa grasa (\%) pliegues corporales (mm, bicipital, tricipital, subescapular, suprailiaco) y presión arterial (mmHg, sistólica y diastólica). En cada periodo se evaluó el peso utilizando una báscula digital (FOSET, modelo BASC-180B, China), la talla con un estadímetro de pared (Zaude 204, China), el índice de masa corporal $\left(\mathrm{IMC}=\mathrm{Peso}, \mathrm{kg} / \mathrm{Talla}, \mathrm{m}^{2}\right)$, la circunferencia de cadera, cintura y brazo con una cinta metálica (Lufkin ExecutiveThinlen, W606ME, USA). Además, se determinó el cambio en la presión arterial sistólica y diastólica con un monitor de presión arterial de muñeca (OMRO, HEM-6221, China), la masa grasa se determinó a partir de los pliegues cutáneos tricipital, bicipital, subescapular y suprailíaco utilizando un plicómetro Slim Guide. Se realizaron comparaciones antes y después de cada periodo de evaluación en cada grupo de recomendación nutricional. En el estudio se cumplió con los principios éticos de las investigaciones en humanos establecidos en la Declaración de Helsinki y de acuerdo al Reglamento de la Ley General de Salud en Materia de Investigación para la Salud.

Análisis estadístico de los datos

Los datos de las variables continuas se representaron como la media \pm desviación estándar y en las variables categóricas se utilizó la frecuencia absoluta y el porcentaje $(n=9)$. La prueba Chi-cuadrada $\left(\chi^{2}\right)$ se utilizó para ver la asociación entre variables categóricas. Para comparar los cambios en las variables de estudio en respuesta a los periodos de las dietas, se utilizaron pruebas $\mathrm{t}$ student para una muestra emparejada. Además, se probaron los cambios entre las dietas en cada periodo utilizando una prueba $t$ student para muestras independientes. Finalmente, se realizó una prueba t student para comparar las diferencias entre los dos grupos pareados. Para el análisis de los datos se utilizó el programa STATISTICA versión 12 (Statsoft, Inc 1984-2014, Tulsa, USA) y Epidat 4.1, con un nivel de confianza del $95 \%$.

\section{Resultados}

Características de los participantes

El presente estudio evaluó los efectos de la incorporación de alimentos bajos en carbohidratos simples y antioxidantes sobre la antropometría en 
mujeres con sobrepeso. Previo a la implementación del cambio en la dieta, a las participantes se les preguntaron características sociales y antecedentes patológicos heredofamiliares. De las nueve participantes, 8 eran trabajadoras fuera del hogar, solo una mostró escolaridad máxima de nivel licenciatura. Cuatro participantes eran casadas y cuatro solteras. Además, dos de ellas mencionaron la presencia de diabetes mellitus e hipertensión y solo una mencionó la obesidad como antecedentes patológicos familiares.

Cambios en las variables evaluadas

Los valores de peso, índice de masa corporal (IMC), circunferencia de cintura y cadera de las participantes antes y después de la aplicación de la dieta baja en carbohidratos simples-antioxidante (BCS-AOX) y la dieta normocalórica se presentan en la Figura 2. Después de un mes de iniciación de ambas dietas, los participantes alcanzaron una reducción significativa del peso y del IMC $(\mathrm{p}<0.05)$. Para la dieta BCS-AOX se encontró una diferencia de $1.56 \mathrm{~kg}$ y en la dieta normocalórica se encontró una diferencia de $0.97 \mathrm{~kg}$ entre el periodo basal y el periodo después de iniciarse las dietas. Con respecto al IMC las dietas presentaron diferencias $(\Delta$ basal- final $)$ de 0.59 y 0.37 $\mathrm{Kg} / \mathrm{m}^{2}$ para BCS-AOX y normocalórica, respectivamente. Esto sugiere que BCS-AOX obtuvo mayor reducción del peso corporal y del IMC respecto a la dieta normal. En la dieta normocalórica los valores obtenidos presentaron una diferencia de 0.72 y $1.33 \mathrm{~cm}$ de cadera y cintura, sin cambio significativo $(p>0.05)$. Sin embargo, los valores de las diferencias para la dieta BCS-AOX fueron de $0.94 \mathrm{~cm}$ para circunferencia de cadera y $1.83 \mathrm{~cm}$ para la de cintura, mostrando diferencia estadística entre los periodos basal y final $(\mathrm{p}<0.05)$. Cabe señalar que no se encontraron diferencias significativas en ninguna de las variables entre la dieta experimental contra la normal al final de cada periodo ( $\mathrm{p}>0.05)$.
Figura 2. Cambios en el peso, índice de masa corporal, circunferencia de cintura y de cadera durante un mes de seguimiento de la dieta baja en carbohidratos simples y alta en antioxidantes (BCS-AOX) y la dieta normal (normocalórica) en mujeres con sobrepeso de Jalcocotán, Nayarit.

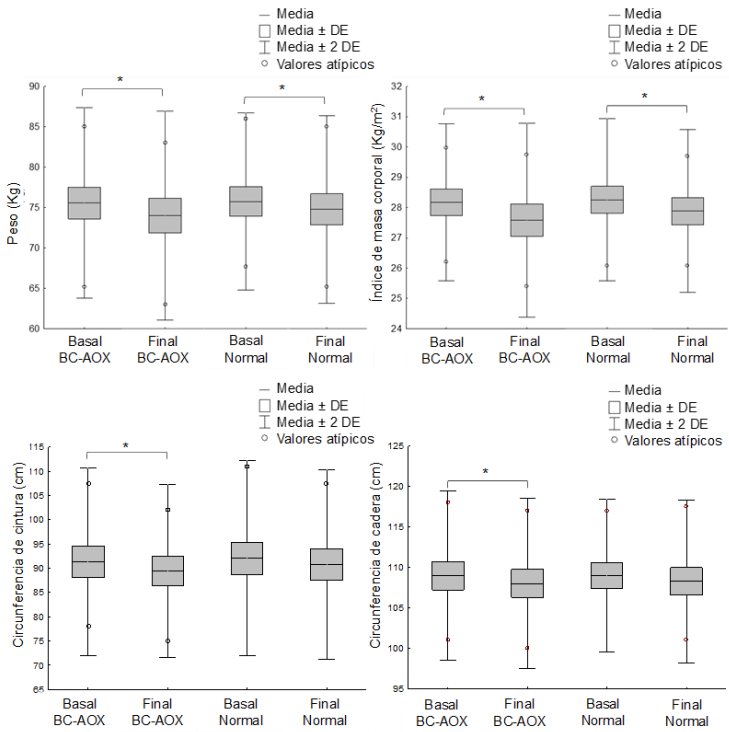

*Indica diferencia estadística para la comparación entre los periodos basal y final para las dietas experimentales utilizando la prueba t pareada $(\mathrm{p}<0.05)$.

En la tabla 1 se muestran los cambios en los parámetros antropométricos y presión arterial de los pacientes antes y después del programa dietético basado en una dieta baja en carbohidratos y antioxidantes (BCS-AOX). La circunferencia de brazo antes de la dieta BCS-AOX fue de $36.05 \mathrm{~cm}$ y se redujo a $35.11 \mathrm{~cm}$. Esta reducción fue estadísticamente significativa $(p<0.05)$. Por otro lado, los valores de la masa grasa, pliegue bicipital, tricipital, subescapular y suprailiaco no mostraron diferencias significativas entre el periodo basal y el final. Sin embargo, la presión diastólica mostró un incremento al final del periodo de evaluación con una diferencia de $6.22 \mathrm{mmHg}(\mathrm{p}<0.05)$. Es importante señalar que a pesar del incremento los valores se encontraron en el rango normal. Por otro lado, tampoco se encontraron diferencias significativas en ninguna de las variables entre las dietas por cada periodo de evaluación $(\mathrm{p}>0.05)$. 
Tabla 1 Evaluación de la circunferencia de brazo, masa grasa, pliegues corporales y presión arterial durante un mes de nutricional utilizando una dieta baja en carbohidratos simples $y$ alta en antioxidantes (BC-AOX) en mujeres con sobrepeso de Jalcocotán, Nayarit. ${ }^{1}$

\begin{tabular}{lcc}
\hline & \multicolumn{2}{c}{ Dieta BC-AOX } \\
\cline { 2 - 3 } & \multicolumn{1}{c}{ Basal } & Final \\
\hline Circunferencia de brazo $(\mathrm{cm})^{*}$ & $36.06 \pm 2.83$ & $35.11 \pm 3.44$ \\
Masa grasa (\%) & $26.03 \pm 3.16$ & $25.46 \pm 3.5$ \\
Pliegues corporales $(\mathrm{mm})$ & & \\
Bicipital & $12.89 \pm 2.57$ & $12.44 \pm 3.13$ \\
Tricipital & $21.78 \pm 4.52$ & $22.33 \pm 2.92$ \\
Subescapular & $23.89 \pm 4.62$ & $24.11 \pm 6.17$ \\
Suprailiaco & $23.44 \pm 5.08$ & $22.56 \pm 5.2$ \\
Presión arterial (mmHg) & $116.44 \pm 8.31$ & $123.56 \pm 7.62$ \\
Sistólica & $74.67 \pm 4.27$ & $80.89 \pm 5.56$ \\
Diastólica* & & \\
\hline 1 Los valores representan la media \pm desviación estándar ( $\mathrm{n}=9$ ). ${ }^{2}$ El \\
valor p fue calculado para la comparación entre los periodos basal \\
y final en las dietas experimentales utilizando la prueba t pareada. \\
* p<0.05
\end{tabular}

En la Tabla 2 se muestran los efectos de la dieta normocalórica sobre los parámetros antropométricos y la presión arterial. No se encontraron cambios significativos en las variables estudiadas $(\mathrm{p}>0.05)$. La circunferencia de brazo promedio fue de 37.78 $\mathrm{cm}$, la masa grasa fue de $25.9 \%$ y los pliegues, bicipital, tricipital, subescapular y suprailiaco presentaron valores de $12.89,21.22,24.56$ y 23.11 $\mathrm{mm}$, respectivamente. La presión arterial sistólica y diastólica se mantuvo constante con valores finales de 121.67 y $78.56 \mathrm{mmHg}$, respectivamente.
Tabla 2. Evaluación de la circunferencia de brazo, masa grasa, pliegues corporales y presión arterial durante un mes de control nutricional utilizando una dieta normal (normocalórica) en mujeres con sobrepeso de Jalcocotán, Nayarit. ${ }^{1}$

\begin{tabular}{|c|c|c|}
\hline & \multicolumn{2}{|c|}{ Dieta Normal } \\
\hline & Basal & Final \\
\hline Circunferencia de brazo $(\mathrm{cm})$ & $35.78 \pm 3.16$ & $35.78 \pm 2.91$ \\
\hline Masa grasa (\%) & $26.86 \pm 2.52$ & $25.9 \pm 3.31$ \\
\hline \multicolumn{3}{|l|}{ Pliegues corporales ( $\mathrm{mm})$} \\
\hline Bicipital & $12.94 \pm 2.65$ & $12.89 \pm 3.26$ \\
\hline Tricipital & $23.22 \pm 2.77$ & $21.22 \pm 2.39$ \\
\hline Subescapular & $24.89 \pm 4.34$ & $24.56 \pm 4.67$ \\
\hline Suprailiaco & $26.22 \pm 5.36$ & $23.11 \pm 4.62$ \\
\hline \multicolumn{3}{|l|}{ Presión arterial $(\mathrm{mmHg})$} \\
\hline Sistólica & $119.56 \pm 9.7$ & $121.67 \pm 12.61$ \\
\hline Diastólica & $77.11 \pm 7.87$ & $78.56 \pm 6.06$ \\
\hline
\end{tabular}

Para determinar los efectos de las dietas sobre los parámetros antropométricos se realizó un análisis comparando las diferencias entre de los periodos por grupos (Tabla 3). Se observó que la diferencia entre los grupos fue significativa en la circunferencia de brazo y el pliegue tricipital $(\mathrm{p}<0.05)$, encontrando que le grupo antioxidante disminuye la circunferencia de brazo, pero el grupo control tiene un mayor efecto en la disminución del pliegue tricipital. También se destacó que la diferencia es mayor en el peso, y el IMC y la presión arterial para BCS-AOX comparado con la dieta normocalorica, sin embargo, estos cambios no fueron estadísticamente significativos $(\mathrm{p}>0.05)$ 
Tabla 3. Análisis de las diferencias por grupo en los parámetros antropométricos en mujeres con sobrepeso. ${ }^{1}$

\begin{tabular}{|c|c|c|c|}
\hline \multirow[t]{2}{*}{ Parámetros } & \multicolumn{2}{|c|}{$\begin{array}{l}\Delta \text { periodos } \\
\text { (antes-después) }\end{array}$} & $\Delta$ grupos \\
\hline & \multicolumn{3}{|c|}{ BCS-AOX Normocalórico } \\
\hline Peso (kg) & 1.57 & 0.98 & 0.59 \\
\hline IMC (km2) & 0.59 & 0.38 & 0.21 \\
\hline Circunferencia de cintura $(\mathrm{cm})$ & 1.83 & 1.33 & 0.50 \\
\hline Circunferencia de cadera $(\mathrm{cm})$ & 0.94 & 0.72 & 0.22 \\
\hline Circunferencia de brazo $(\mathrm{cm})^{*}$ & 0.94 & 0 & 0.94 \\
\hline Presión sistólica (mmHg) & -7.11 & -2.11 & -5.00 \\
\hline Presión diastólica (mmHg) & -6.22 & -1.44 & -4.78 \\
\hline Masa grasa $(\%)$ & 0.56 & 0.96 & -0.39 \\
\hline Pliegue bicipita(mm) & 0.44 & 0.06 & 0.39 \\
\hline Pliegue tricipital (mm)* & -0.56 & 2 & -2.56 \\
\hline Pliegue Subescapular (mm) & -0.22 & 0.33 & -0.56 \\
\hline Pliegue Suprailíaco (mm) & 0.89 & 3.11 & -2.22 \\
\hline \multicolumn{4}{|c|}{${ }^{1}$ Los valores representan la media de las diferencias por periodos y por grupo. } \\
\hline \multicolumn{4}{|c|}{$\begin{array}{l}{ }^{2} \text { El valor p fue calculado para la comparación entre las diferencias obtenidas del } \\
\text { periodo inicial y final entre los grupos utilizando la prueba t para la diferencia de } \\
\text { dos grupos pareados. } \\
{ }^{*} p<0.05\end{array}$} \\
\hline
\end{tabular}

\section{Discusión}

En el presente trabajo se evaluó el efecto de la sustitución de alimentos antioxidantes bajos en carbohidratos simples sobre las características corporales de mujeres con sobrespeso. En este sentido, se observaron disminuciones significativas del peso, e IMC en la dieta normocalórica y en la dieta con alimentos bajos en carbohidratos y altos en antioxidantes (BCS-AOX), pero solo se observó una disminución en los valores de circunferencia de cintura y cadera para esta última dieta. En este sentido, Annuzzi et al. (2014) no encontraron cambios significativos en el peso y la circunferencia de cintura después de ocho semanas de administración de una dieta naturalmente enriquecida con polifenoles $(-0.57 \mathrm{~kg}$ y $-0.6 \mathrm{~cm})$ con respecto a una dieta isocalórica $(-0.11 \mathrm{~kg} \mathrm{y}-0.8 \mathrm{~cm})$ en adultos con riesgo cardiovascular. Recientemente se ha informado que la diferencia media en la pérdida peso oscila entre $-0.98 \mathrm{y}-7.05 \mathrm{~kg}$ para las dietas bajas en carbohidratos y entre $-1.75 \mathrm{y}-2.24 \mathrm{~kg}$ para la dieta mediterránea (Dinu et al., 2020). Cabe señalar que la diferencia en el peso obtenido para la dieta BCS-AOX $(1.56 \mathrm{~kg})$ se encuentra entre los valores reportados para las dietas bajas en carbohidratos. Los ligeros cambios en los parámetros antropométricos podrían atribuirse a la ingesta de fibra dietética y antioxidantes de las verduras y frutas presentes en mayor cantidad en la dieta BCS-AOX. En este sentido, Vega et al. (2019), quienes realizaron una intervención basada en el incremento en el consumo de verduras y fruta, observaron una disminución de la prevalencia de sobrepeso y obesidad en adolescentes mexicanos de $13.8 \%$ a $6.1 \%$. No obstante, la evidencia no es contundente, ya que autores como Ruiz-Montero (2016) mencionan que es la combinación de diferentes factores alimentarios como una dieta rica en verduras, fruta y el control en la ingesta de carne roja lo que podría a ayudar en la disminución del IMC. En este trabajo no se encontraron cambios significativos en los parámetros antropométricos, sin embargo, la modificación en los alimentos consumidos podría mejorar el perfil antioxidante en los individuos, sin embargo, es necesario incluir análisis bioquímicos en la investigación. En este sentido, Crujeiras et al. (2006) administraron una dieta con restricción de calorías $(600 \mathrm{kcal})$ y sustituyeron $5 \%$ (control) y $15 \%$ de la energía total por frutas. Los autores no encontraron cambios entre el grupo de intervención y el grupo control con respecto a la pérdida de peso pero encontraron una disminución (-30\%) de la concentración de malondialdehído atribuyendo un efecto antioxidante asociado a la fibra dietética (Crujeiras et al., 2006). Es bien conocido el beneficio del consumo de fibra dietética sobre todo de las fibras solubles. Las fibras solubles (como los $\beta$-glucanos) influyen en el apetito a través de propiedades químicas y físicas y los posibles mecanismos para el efecto saciante incluyen la naturaleza viscosa en el tracto gastrointestinal o mediante la prolongación de la elevación de la colecistoquinina (supresores del apetito) que resulta en una saciedad prolongada (Fuller et al., 2016). Por otro lado, los polifenoles son bien conocidos por brindar beneficios para la salud, incluidas acciones antihipertensivas a través de acciones antiinflamatorias y antioxidantes. Un estudio en humanos con síndrome metabólico ha informado que la ingesta de quercetina de $150 \mathrm{mg}$ / día durante cinco semanas disminuye la presión arterial sistólica (Egert et al., 2009). Estos resultados no coinciden con lo reportado en este trabajo donde se observó un incremento en la presión sistólicas con la dieta BCSAOX, sin embargo, estas diferencias pueden deberse al estado patológico de los pacientes. En el presente trabajo las participantes solo presentaron problemas de sobrepeso y ninguna otra patología agregada. En este sentido, Edwards et al. (2007) indicaron que la ingesta de quercetina de $730 \mathrm{mg}$ / día durante cuatro semanas disminuye la presión arterial sistólica y diastólica en pacientes hipertensos en etapa 1, pero no tiene efectos sobre la presión arterial sistólica o 
diastólica con pre hipertensión. En la tabla 3 se observan una mayor reducción del peso y el índice de masa corporal para BCS-AOX, aunque no fue estadísticamente significativa. Sin embargo, los resultados sugieren que la incorporación a la dieta de alimentos bajos en carbohidratos y con propiedades antioxidantes modifican los parámetros antropométricos. La uva es un alimento reconocido por su poder antioxidante. Según Chuang y McIntosh (2011) los mecanismos potenciales por los cuales las uvas ricas en polifenoles previenen la inflamación mediada por la obesidad son la expresión de genes o proteínas antioxidantes, la atenuación de la señalización del estrés del retículo endoplásmico, el bloqueo de las citocinas proinflamatorias, la supresión de la expresión de genes inflamatorios y la inducción del metabolismo mediante el aumento de la actividad de la histona desacetilasa. Sin duda, el efecto de los fitoquímicos de las plantas es tan relevante que incluso se han realizados estudios como el de Tripp et al. (2019) donde se confirmó que la adición de suplementos nutracéuticos específicos a una dieta mediterránea restringida en calorías con modificaciones en el estilo de vida mejora múltiples factores de riesgo de longevidad de manera más efectiva que la modificación de la dieta y el estilo de vida solo. Por último, es importante considerar que el tamaño de la muestra, la falta de análisis bioquímicos y el corto periodo de la intervención podrían ser limitantes en los hallazgos de este estudio y deben ser consideradas para determinar los efectos que el cambio en el perfil de alimentos proporciona a la salud de los individuos.

\section{Conclusiones}

En este trabajo se estableció que la sustitución en un $25 \%$ de la ingesta calórica total de alimentos bajos en carbohidratos y altos en antioxidantes a la dieta modificó favorablemente los parámetros antropométricos de la misma forma que lo hace una dieta normocalórica en mujeres con sobrepeso que asisten al gimnasio municipal de Jalcocotán Nayarit durante un mes de intervención. Los alimentos incorporados pueden proporcionar mayor cantidad de fibra dietética y polifenoles, los cuales podrían representar un papel clave en el tratamiento dietético y control del sobrepeso y obesidad. Sin embargo, son necesario estudios controlados donde se evalúen marcadores bioquímicos en comunidades específicas del estado de Nayarit para fortalecer las evidencias encontradas en este esta investigación.

\section{Agradecimientos}

Arteaga-Murguia, DM, agradece al Sr. Jesús Arteaga, Sra. Emilia Murguia, Carlos Arvizu, Aliz Arvizu, a la Sra. Amparo Escobedo, así como a cada participante por su amable colaboración en la realización de este proyecto.

\section{Bibliografía}

Aguiar-Bloemer, A. C., Japur, C. C., Francisco, L. V., y Diez-Garcia, R. W. (2019). Dietary quality differences between women with and without weight loss in nutritional treatment. Clinical nutrition ESPEN, 34, 110-115.

Amirkhizi, F., Siassi, F., Minaie, S., Djalali, M., Rahimi, A., y Chamari, M. (2010). Is obesity associated with increased plasma lipid peroxidation and oxidative stress in women? Atherosclerosis, 2(4). http://www.arya.mui.ac.ir/index.php/arya/article /view/165

Annuzzi, G., Bozzetto, L., Costabile, G., Giacco, R., Mangione, A., Anniballi, G., Vitale, M., Vetrani, C., Cipriano, P., y Corte, G. D. (2014). Diets naturally rich in polyphenols improve fasting and postprandial dyslipidemia and reduce oxidative stress: a randomized controlled trial. The American journal of clinical nutrition, 99(3), 463-471.

Barrera-Reyes, P., Hernández-Ramírez, N., Cortés, J., Poquet, L., Redeuil, K., Rangel-Escareño, C., Kussmann, M., Silva-Zolezzi, I., y Tejero, M. (2019). Gene expression changes by highpolyphenols cocoa powder intake: a randomized crossover clinical study. European journal of nutrition, 58(5), 1887-1898.

Chuang, C.-C., y McIntosh, M. (2011). Potential mechanisms by which polyphenol-rich grapes prevent obesity-mediated inflammation and metabolic diseases. Annual review of nutrition, $31,155-176$.

Crujeiras, A. B., Parra, M. D., Rodríguez, M. C., de Morentin, B. E. M., y Martínez, J. A. (2006). A role for fruit content in energy-restricted diets in improving antioxidant status in obese women during weight loss. Nutrition, 22(6), 593-599. 
Dinu, M., Pagliai, G., Angelino, D., Rosi, A., Dall'Asta, M., Bresciani, L., Ferraris, C., Guglielmetti, M., Godos, J., y Del Bo, C. (2020). Effects of popular diets on anthropometric and cardiometabolic parameters: An umbrella review of meta-analyses of randomized controlled trials. Advances in Nutrition, 11(2), 815-833.

Dosamantes-Carrasco, L. D., Méndez-Hernández, P., Flores, Y. N., Siani, C., Denova-Gutiérrez, E., Gallegos-Carrillo, K., Ramírez, P., RiveraParedez, B., Salazar-Martínez, E., y Salmerón, J. (2017). Influence of mealtime habits on the risk of weight gain and obesity in Mexican adults. Public health nutrition, 20(2), 220-232.

Dreher, M. L., y Ford, N. A. (2020). A Comprehensive Critical Assessment of Increased Fruit and Vegetable Intake on Weight Loss in Women. Nutrients, 12(7), 1919.

Edwards, R. L., Lyon, T., Litwin, S. E., Rabovsky, A., Symons, J. D., y Jalili, T. (2007). Quercetin reduces blood pressure in hypertensive subjects. The Journal of nutrition, 137(11), 2405-2411. https://academic.oup.com/jn/article/137/11/2405 $\underline{14750737}$

Egert, S., Bosy-Westphal, A., Seiberl, J., Kürbitz, C., Settler, U., Plachta-Danielzik, S., Wagner, A. E., Frank, J., Schrezenmeir, J., y Rimbach, G. (2009). Quercetin reduces systolic blood pressure and plasma oxidised low-density lipoprotein concentrations in overweight subjects with a high-cardiovascular disease risk phenotype: a double-blinded, placebo-controlled cross-over study. British Journal of Nutrition, 102(7), 10651074. https://doi.org/10.1017/S0007114509359127

Fuller, S., Beck, E., Salman, H., y Tapsell, L. (2016). New horizons for the study of dietary fiber and health: a review. Plant foods for human nutrition, 71(1), $1-12$. https://link.springer.com/article/10.1007/s11130 $\underline{-016-0529-6}$

INSP. (2012). Encuesta Nacional de Salud y Nutrición - 2012. Resultados por entidad federativa, Nayarit. Consultado de: https://ensanut.insp.mx/encuestas/ensanut2012/d
octos/informes/Nayarit-OCT.pdf. Secretaría de Salud, Instituto Nacional de Salud Pública., 1-42.

INSP. (2018). Encuesta Nacional de Salud y Nutrición - 2018. Informe de Resultados, Consultado de: https://ensanut.insp.mx/encuestas/ensanut2018/i nformes.php. Secretaría de Salud, Instituto Nacional de Salud Pública., 1-42.

Marseglia, L., Manti, S., D’Angelo, G., Nicotera, A., Parisi, E., Di Rosa, G., Gitto, E., y Arrigo, T. (2015). Oxidative stress in obesity: a critical component in human diseases. International journal of molecular sciences, 16(1), 378-400. https://www.mdpi.com/1422-0067/16/1/378

Pérez-Lizaur, A., Palacios-González, B., CastroBecerra, A., y Flores-Galicia, I. (2014). SMAE: Sistema Mexicano de Alimentos Equivalentes. México, Fomento de Nutrición y Salud.

Pérez-Tepayo, S., Rodríguez-Ramírez, S., UnarMunguía, M., y Shamah-Levy, T. (2020). Trends in the dietary patterns of Mexican adults by sociodemographic characteristics. Nutrition Journal, 19, 1-10.

Roengrit, T., Wannanon, P., Prasertsri, P., Kanpetta, Y., Sripanidkulchai, B.-o., Wattanathorn, J., y Leelayuwat, N. (2015). Antioxidant effect of Phyllanthus amarus after moderate-intensity exercise in sedentary males: a randomized crossover (double-blind) study. Journal of physical therapy science, 27(4), 1181-1186.

Roza, A. M., y Shizgal, H. M. (1984). The Harris Benedict equation reevaluated: resting energy requirements and the body cell mass. American journal of clinical nutrition, 40(1), 168-182. https://academic.oup.com/ajcn/articleabstract/40/1/168/4691315

Ruiz-Montero, P. J. (2016). 8. Ingesta dietética y actividad física en el tiempo libre de adultasmayores en ámbito rural mediterráneo. Archivos Latinoamericanos de Nutrición, 66(4).

Slavin, J., y Green, H. (2007). Dietary fibre and satiety. Nutrition Bulletin, 32, 32-42. 
https://onlinelibrary.wiley.com/doi/full/10.1111/ j.1467-3010.2007.00603.X

Suverza, A., y Haua, K. (2010). ABCD de la evaluación del estado de nutrición. México, DF: McGraw-Hill.

Svendsen, M., Blomhoff, R., Holme, I., y Tonstad, S. (2007). The effect of an increased intake of vegetables and fruit on weight loss, blood pressure and antioxidant defense in subjects with sleep related breathing disorders. European journal of clinical nutrition, 61(11), 1301-1311.

Tripp, M. L., Dahlberg, C. J., Eliason, S., Lamb, J. J., Ou, J. J., Gao, W., Bhandari, J., Graham, D., Dudleenamjil, E., y Babish, J. G. (2019). A LowGlycemic, Mediterranean Diet and Lifestyle Modification Program with Targeted Nutraceuticals Reduces Body Weight, Improves Cardiometabolic Variables and Longevity Biomarkers in Overweight Subjects: A 13-Week Observational Trial. Journal of Medicinal Food, 22(5), 479-489. https://www.liebertpub.com/doi/abs/10.1089/jmf $\underline{.2018 .0063}$

Tucakovic, L., Colson, N., y Singh, I. (2015). Relationship between common dietary polyphenols and obesity-induced inflammation. Food Public Health, 5(3), 84-91. https://core.ac.uk/download/pdf/143900107.pdf

Vega, S., León, C. C. R. V., Tolentino, R. G., y Radilla, M. J. R. E. N. C. (2019). Intervención para la incentivación del consumo de verduras y frutas como estrategia para la disminución del exceso de peso en adolescentes de la Ciudad de México. Revista Española de Nutrición Comunitaria, 25(1). http://www.renc.es/imagenes/auxiliar/files/REN C 2019 1 02. CC Radilla. Incremento en el consumo de verduras y frutas.pdf 